\title{
Complexity emerges from chemistry
}

\author{
Chemical systems can show complex behaviour that is not seen in individual molecules or reactions. Helena $\mathrm{S}$. \\ Azevedo, Sarah L. Perry, Peter A. Korevaar, and Dibyendu Das report on the emergence of this complex behaviour, \\ which was discussed at the Virtual Symposium on Systems Chemistry
}

ystems chemistry is a new frontier in molecular sciences, focusing on (multi-)functional behaviour that emerges from ensembles of molecular components $^{1-6}$. Creating systems of molecules can establish behaviour that goes beyond the sum of the individual elements - while taking advantage of well-established methods and traditional chemistry approaches for the study of individual molecules and reactions. The most striking example of such emerging behaviour is life itself, and unravelling the rules involves decreasing the gap between 'dead' molecules and living matter. While still in its infancy, this exciting challenge has drawn the attention from chemists worldwide, with backgrounds varying from supramolecular chemistry to prebiotic chemistry, biochemistry, theoretical biology, and soft matter.

Virtual Symposium on Systems Chemistry was hosted by Advanced Science Research Center (CUNY) and organized by Gonen Ashkenasy, Rafal Klajn, David Lynn, Sijbren Otto, Rebecca Schulman and Rein Ulijn from 18-20 May 2020. The discussions covered how concepts such as supramolecular assembly, out-of-equilibrium, chemical networks, and compartmentalization translate into phenomena that vary from self-organization to motion, functional (bio)materials, new insights into the origins of life, and intervention with the SARS-CoV-2 virus responsible for the COVID-19 pandemic (the main reason for the online format of the symposium, and, ironically, a supramolecular system that in itself has many aspects related to systems chemistry) (Fig. 1).

The meeting began with Nobel Laureate Ben Feringa (University of Groningen), who presented the challenge of integrating motion - a hallmark of life - into molecular systems. Starting off with the photoisomerization of a core double bond in a chiral molecule, his group developed a motor that operates at the nanoscale but produces, by amplification of collective molecular motion, motility at much larger scales. Molecular motors were shown to generate rotation inside metal-organic

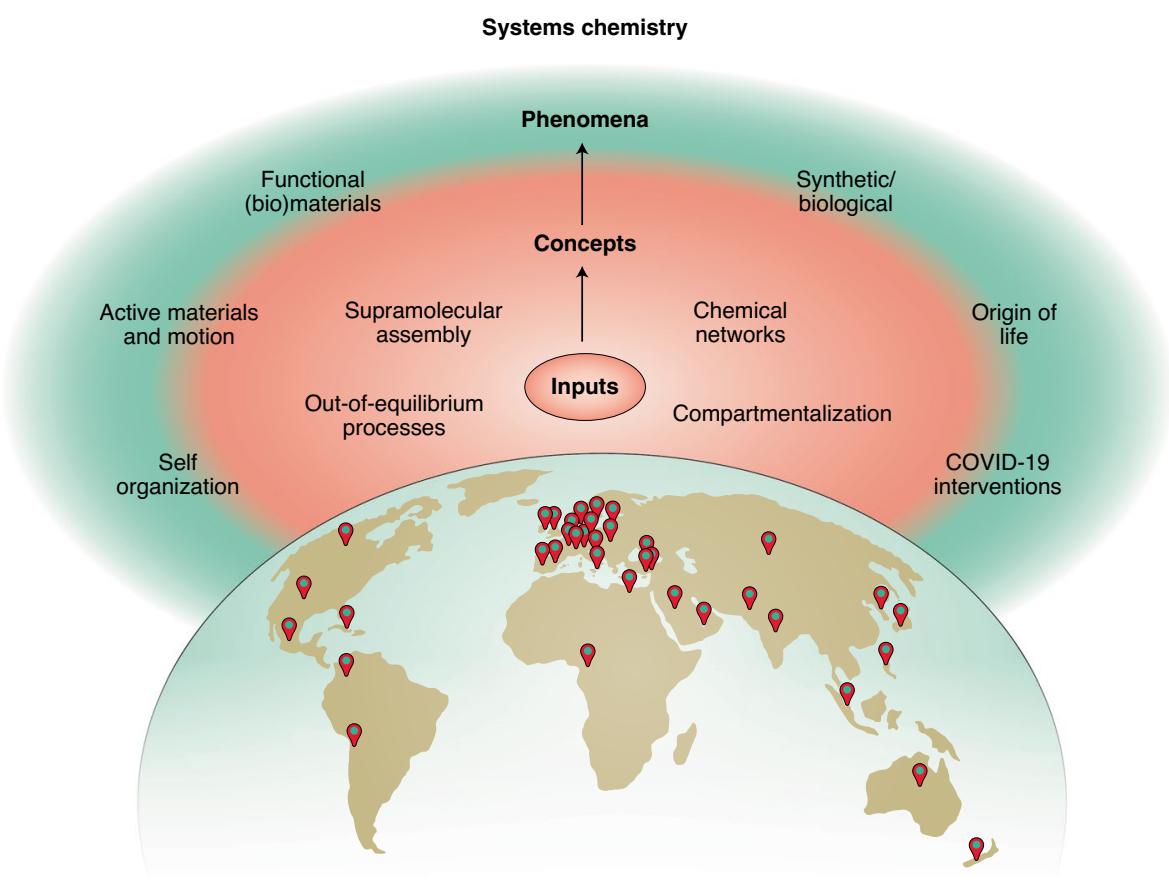

Fig. 1 | Virtual Symposium on Systems Chemistry. The symposium discussed how chemical inputs, concepts, and phenomena drive the emerging field of systems chemistry.

frameworks, actuate muscle-like hydrogels and direct the differentiation of stem cells at adsorbed protein layers: exemplifying how well-orchestrated motion from molecular systems emerges into systems with potential catalytic, self-healing, or therapeutic functions.

Another hallmark of life is self-organization of matter into patterns and structures. Petra Schwille (MPI Biochemistry) showed the pathways underlying the emergence of patterns from the Min protein system that serves to localize the cell centre during the division of E. coli. Reducing the complexity of the system reveals key network motifs enabling pattern formation; however, at the same time, complexity might be key to the robustness of pattern-forming systems in life. In a more synthetic context, Peter Korevaar (Radboud University) demonstrated the emergence of multi-droplet self-organization at air-water interfaces, sustained by the Marangoni flow between amphiphile source and drain droplets that are in turn kept in position by filaments assembled from said amphiphile.

Self-organization critically relies on the controlled motion of the entities involved. Lauren Zarzar (Pennsylvania State University) developed a system of oil-in-water microdroplets that chased each other in a predator-prey analogy. This motion is powered by the free energy of mixing, regulated by a unique chemotactic signalling between the droplets. Daphne Klotsa (University of North Carolina at Chapel Hill) used computational simulations to demonstrate how swarms of active matter particles show emergent collective behaviour in fluids, directed by many-body hydrodynamic interactions. Ayusman Sen (Pennsylvania State University) showed motion on a much smaller scale with collective behaviour of molecules that could be facilitated by cascade catalysis and enzyme proximity. He further showed how chemotaxis can allow selective, time 
dependent, and sequential assembly of catalyst particles.

The out-of-equilibrium nature of active matter can often be controlled by well-regulated dissipative mechanisms. Dibyendu Das (IISER Kolkata) highlighted the importance of intrinsic negative feedback to regulate such out-of-equilibrium behaviour in autonomous materials. Synthetic microtubule analogues were shown to stop their own formation via accelerated catalytic degradation of the building blocks.

In addition to motion, self-reproduction is another aspect of synthetic biology that requires encoding and translation of information. Using microfluidics and an experimental model of synthetic ribozymes that autocatalyse their own formation from RNA oligomers, Phillipe Nghe (ESPCI Paris) created a combinatorial process to investigate reproduction and diversity in these autocatalytic networks. He identified design rules for developing chemical systems with evolutionary characteristics, having implications for origin-of-life research. Working to create a simplified system based on biology, Hannes Mutschler (MPI Biochemistry) discussed the generation of a cell-free, partially self-replicating in vitro translation system that can mimic the essential features of living matter.

Many living or bioinspired systems use self-assembly to create compartments that store and exchange chemicals, and facilitate chemical reactions. While amphiphiles such as surfactants and lipids provide the most direct parallels to cells, Sarah Perry (University of Massachusetts Amherst) explained how association between oppositely-charged macro-ions, such as polymers, proteins, and micelles, provides liquid-liquid phase separation (that is, complex coacervation) as an alternative strategy to create compartments. Complex coacervation has a long history in protocell models, and has more recently been observed in 'membraneless organelles' that enable compartmentalization without the presence of an impermeable membrane barrier. Stephen Mann (University of Bristol) demonstrated the potential of protocells towards distributed computation in compartmentalized microscale objects, based on DNA-based signal processing and enzyme-mediated interactivity and feedback. This compartmentalization enables, as Mann argued, a modular design in the engineering of systems with a reductionist approach. Pall Thordarson (UNSW Sydney) spoke further on compartmentalization, where the use of polymer amphiphiles to create membrane-mimicking polymersomes allows for the creation of non-spherical structures that have utility in applications such as drug delivery.

Self-assembled systems based on natural building blocks can also be used as structural and functional components for (bio)materials science and nanotechnology. Helena Azevedo (Queen Mary University of London) shared her work on molecularly engineered peptide nanomaterials as model substrates to unravel the mechanism of enzyme catalysis and how biocatalysis can be applied to reveal the molecular dynamics in self-assembled systems. Jen Heemstra (Emory University) talked about 'bilingual' biopolymers that bridge peptides and nucleic acids to access materials that can encode information, create compartments, and facilitate intracellular delivery.

Inevitably, the virtual event had a session on COVID-19 where Sam Gellman (University of Wisconsin-Madison) and Pall Thordarson shared their efforts in combating the spread of coronavirus infection. Gellman discussed the development of backbone modified peptides that can act as inhibitors against COVID19. These helical $\alpha / \beta$ peptides resisted degradation by viral proteases and were thought to interact with the spike protein of the virus. In a powerful demonstration of the effectiveness of scientific outreach, Thordarson described how his Twitter thread explaining the importance of soap in neutralising the coronavirus went 'viral' - a brilliant lesson on the chemistry and nanoscience of soap and viruses.

An unspoken challenge in science that has been exacerbated by the physical distancing requirements of the COVID-19 pandemic is mental health. Jen Heemstra addressed the challenges, such as imposter syndrome and the stress of joining a new program, and suggested strategies, such as changing the stories that we tell ourselves, which could help both generally and during the current crisis.

Ultimately, this virtual symposium brought together more than 550 participants from 46 countries, five continents, and a huge spread of time zones. The Twitter poster session enjoyed $>63,000$ engagements, with an average of $>600$ per poster, and an interactive Slack forum facilitated the critical networking discussions, which are the heart of many conference experiences.

While the COVID-19 pandemic may have forced many of us to physically distance, the success of this virtual event can serve as a model for overcoming many of the practical and financial barriers for conference travel in the future. At the same time, it exemplifies how systems chemistry can unite a variety of areas ranging from chemical networks to assembling and active matter, to explore emergent, synergistic phenomena that will address new problems in the years to come.

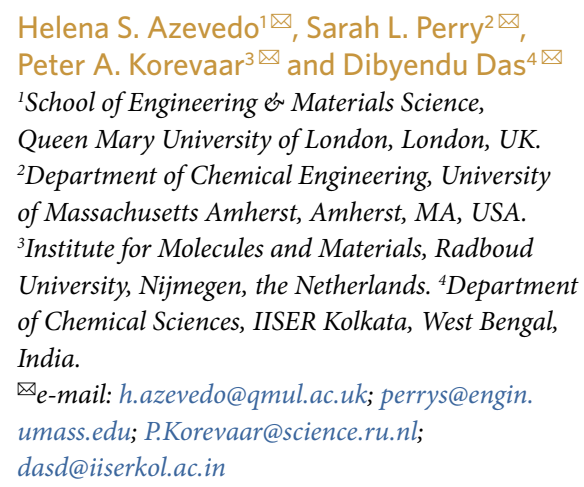

Published online: 17 August 2020

https://doi.org/10.1038/s41557-020-0537-x

References

1. Mattia, E. \& Otto, S. Nature Nanotechnol. 10, 111-119 (2015).

2. Ashkenasy, G., Hermans, T. M., Otto, S. \& Taylor, A. F. Chem. So Rev. 46, 2543-2554 (2017)

3. Kroiss, D., Ashkenasy, G., Braunschweig, A. B., Tuttle, T. \& Ulijn, R. V. Chem. 5, 1917-1920 (2019)

4. Scalise, D. \& Schulman, R. Annu. Rev. Biomed. Eng. 21 469-493 (2019).

5. Bai, Y., Chotera, A., Taran, O., Liang, C., Ashkenasy, G. \& Lynn, D. G. Chem. Soc. Rev. 47, 5444-5456 (2018).

6. van Esch, J. H., Klajn, R. \& Otto, S. Chem. Soc. Rev. 46 5474-5475 (2017) 